\title{
Origin and Evolution of Dendritic Epidermal T Cells
}

\author{
Yoichi Sutoh ${ }^{1}$, Rania Hassan Mohamed ${ }^{2}$ and Masanori Kasahara ${ }^{3 *}$ \\ ${ }^{1}$ Division of Biobank and Data Management, Iwate Tohoku Medical Megabank Organization, Iwate Medical University, \\ Shiwa-gun, Japan, ${ }^{2}$ Department of Biochemistry, Faculty of Science, Ain Shams University, Cairo, Egypt, ${ }^{3}$ Department \\ of Pathology, Faculty of Medicine, Hokkaido University, Sapporo, Japan
}

\section{OPEN ACCESS}

Edited by:

Thomas Herrmann,

Universität Würzburg, Germany

Reviewed by:

Daniel J. Pennington, Queen Mary University of London,

United Kingdom

Julie Marie Jameson,

California State University San Marcos, United States

*Correspondence:

Masanori Kasahara mkasaha@med.hokudai.ac.jp

Specialty section: This article was submitted to T Cell Biology,

a section of the journal

Frontiers in Immunology

Received: 27 February 2018 Accepted: 27 April 2018

Published: 14 May 2018

Citation:

Sutoh Y, Mohamed RH and Kasahara M (2018) Origin and Evolution of Dendritic Epidermal T Cells.

Front. Immunol. 9:1059. doi: 10.3389/fimmu.2018.01059
Dendritic epidermal T cells (DETCs) expressing invariant $\mathrm{V} \gamma 5 \mathrm{~V} \delta 1$ T-cell receptors (TCRs) play a crucial role in maintaining skin homeostasis in mice. When activated, they secrete cytokines, which recruit various immune cells to sites of infection and promote wound healing. Recently, a member of the butyrophilin family, Skint1, expressed specifically in the skin and thymus was identified as a gene required for DETC development in mice. Skint1 is a gene that arose by rodent-specific gene duplication. Consequently, a gene orthologs to mouse Skint1 exists only in rodents, indicating that Skint1-dependent DETCs are unique to rodents. However, dendritic-shaped epidermal $\gamma \delta T$ cells with limited antigen receptor diversity appear to occur in other mammals. Even lampreys, a member of the most primitive class of vertebrates that even lacks TCRs, have $\gamma \delta$ T-like lymphocytes that resemble DETCs. This indicates that species as divergent as mice and lampreys share the needs to have innate-like T cells at their body surface, and that the origin of DETC-like cells is as ancient as that of lymphocytes.

Keywords: dendritic epidermal T cell, $\gamma \delta$ T cell, Skint1, skin, epidermis, intraepithelial lymphocyte

\section{INTRODUCTION}

In the mouse epidermis, $~ 95 \%$ of cells are keratinocytes, and the remaining $5 \%$ are immune cells such as Langerhans cells and T cells. The majority of epidermis-resident T cells are $\gamma \delta \mathrm{T}$ cells with a highly dendritic shape, extending dendrites both basally and apically; along with Langerhans cells, these $\gamma \delta$ $\mathrm{T}$ cells form an interdigitating network within the layers of the epidermis (1). Therefore, they are called dendritic epidermal T cells (DETCs) (2). Interestingly, 90\% of DETCs express an invariant V $\gamma 5 \mathrm{~V} \delta 1$ T-cell receptor (TCR) without any junctional diversity [also called V $\gamma 3 \mathrm{~V} \delta 1$ TCR according to the nomenclature of Garman et al. (3)]. It is thought that DETCs recognize a limited set of "stress antigens" induced on damaged or dysregulated keratinocytes through their invariant TCRs in a major histocompatibility complex (MHC)-independent manner (4). Although the molecular identity of "stress antigens" recognized by V $\gamma 5 \mathrm{~V} \delta 1$ TCR remains unknown, co-stimulatory molecules on DETCs, which synergistically amplify TCR signals, have been identified. The most important among them are the junctional adhesion molecule-like protein JAML (5), CD100 (also known as semaphorin 4D) (6), and NKG2D receptors $(7,8)$, which interact with the coxsackie and adenovirus receptor, plexin B2, and a group of stress-inducible MHC class I-like molecules known as NKG2D ligands $(9,10)$, respectively.

Once activated, DETCs retract their dendrites, adopt a rounded shape, and secrete a range of cytokines, chemokines, and tissue-specific growth factors, leading to increased keratinocyte proliferation and recruitment of infiltrating leukocytes, thereby promoting wound healing and immune surveillance in the skin. Among the cytokines secreted by DETCs is insulin-like growth factor 1, which aids wound healing by preventing apoptosis of keratinocytes and DETCs themselves in an 
autocrine manner (11). Although controversial, one recent study has shown that a subset of DETCs secretes interleukin-17A that induces production of antimicrobial peptides such as $\beta$-defensin 3 and regenerating islet-derived protein $3 \gamma$ (12). The latter peptide also induces keratinocyte proliferation and differentiation after skin injury (13), indicating that interleukin-17 plays a role in both infection control and epithelial proliferation at wound sites.

In the thymus of fetal mice, $\gamma \delta$ T cells with particular $\gamma$ and $\delta$ chains appear sequentially in discrete waves. DETC progenitors bearing V $\gamma 5 \mathrm{~V} \delta 1$ TCRs appear at embryonal days 14-16 $(14,15)$, after which they home to the epidermis. On the other hand, terminal deoxynucleotidyl transferase (TdT), which generates junctional diversity in $\mathrm{V}(\mathrm{D}) \mathrm{J}$ recombination by attaching additional nucleotides (so-called $\mathrm{N}$ nucleotides) at the $3^{\prime}$-end of gene segments in a template-independent way (15), is not expressed in fetal thymus and starts to be expressed 4 days after birth (14). Forced expression of TdT in fetal thymus produces DETCs expressing $\mathrm{V} \gamma 5 \mathrm{~V} \delta 1$ TCRs with junctional diversity, which populate the epidermis of newborn mice (16). These DETCs, however, gradually disappear after birth, unlike normal DETC. Therefore, it appears that TCR specificity is not required for epidermal migration of DETC progenitors, but important for renewing and sustaining DETCs in the epidermis. Likewise, epidermis-resident T cells in TCR $\delta$-deficient mice, which mainly express variable $\alpha \beta$ TCRs, are gradually lost and not retained over a lifetime (17), again indicating the importance of TCR specificity for the maintenance of epidermal T cells.

Dendritic epidermal $\mathrm{T}$ cells have been identified in rodents such as mice $(18)$ and rats $(19,20)$. However, little is known about the origin and evolution of DETCs. Recently, a gene essential for DETC development, named Skint1 (selection and upkeep of intraepithelial T cells protein 1), was identified in mice $(21,22)$. Interestingly, the Skint1-like (SKINT1L) gene is inactivated in humans, raising the possibility that this inactivation might be responsible for the deficiency of DETCs in humans. In this review, we summarize the evolution of the SKINT gene family and its implications for the origin and evolution of DETCs. Available evidence indicates that Skint1-dependent DETCs are unique to rodents. However, if we define DETCs more broadly as dendriticshaped epidermal $\gamma \delta$ T cells with limited antigen receptor diversity, they seem to occur in other mammals. Indeed, even lampreys, a member of the most primitive class of vertebrates equipped with lymphocytes, have DETC-like cells, suggesting that DETCs also exist in jawed vertebrates other than mammals.

\section{SKINT1 AND THE SKINT GENE FAMILY}

The epidermis of FVB/N mice from Taconic Farms (FVB/N Tac) lacks $V \gamma 5 \mathrm{~V} \delta 1$ DETCs, while $\gamma \delta$ T-cell repertoires in other tissues are normal (23). Skint1 was identified as a gene responsible for this depletion of canonical DETCs (21). SKINT1 is a membranebound immunoglobulin (Ig) superfamily protein made up of an Ig variable (IgV) domain, an Ig constant domain and three transmembrane regions. It is specifically expressed by thymic epithelial cells and skin keratinocytes. The Skint 1 gene of FVB/N Tac mice contains a premature termination codon in the region coding for the segment between the second and third transmembrane regions. In Skint1-deficient mice, V $\gamma 5 \mathrm{~V} \delta 1 \mathrm{~T}$ cells are present in fetal thymus in comparable numbers to wild-type FVB/N mice at embryonic days 14-16.5, but the production of mature $\mathrm{V} \gamma 5 \mathrm{~V} \delta 1$ T cells migrating to the epidermis is impaired because of defective thymic selection of $\mathrm{V} \gamma 5 \mathrm{~V} \delta 1 \mathrm{~T}$ cells (23). The complementarity determining region 3-like loop in the IgV domain of SKINT1 molecules appears important for this selection (24). However, it is not known whether SKINT1 or the SKINT1 molecular complex interacts with $\mathrm{V} \gamma 5 \mathrm{~V} \delta 1$ TCR itself or an as yet uncharacterized molecule expressed uniquely on DETC progenitors (22).

Skint1 is a member of the Skint gene family. Mice have 11 Skint genes (paralogs) designated Skint1 to Skint11, coding for structurally related proteins with similar, though not identical, expression patterns (21). These paralogs appear to have distinct functions. Thus, neither Skint2 nor Skint7 can compensate for the loss of Skint1 function in reaggregate fetal thymic organ culture (22). Furthermore, mice selectively deficient in epidermal Skint1 expression show only a minor delay in wound healing compared to mice deficient in Skint3 or Skint9, suggesting that Skint1 is mainly involved in the maturation of DETC progenitors in the thymus, and that Skint3 and Skint9 play a more important role in mediating DETC activation in the epidermis (25). These observations suggest that Skint paralogs have undergone functional specialization, with only Skint1serving as a selecting component for $\mathrm{V} \gamma 5 \mathrm{~V} \delta 1 \mathrm{~T}$ cells.

\section{EVOLUTION OF THE SKINT GENE FAMILY}

The SKINT gene family, which occurs only in placental mammals, is a member of a larger gene family known as the butyrophilin family (26-28). It comprises three subfamilies, SKINT1, SKINT7, and SKINT9 (29). Figure 1 shows the phylogenetic tree of SKINT1 subfamily genes in placental mammals. Whereas mice and rats have multiple copies of these subfamily genes, most mammals have either a single copy of SKINT1 genes known as SKINT1L or altogether lack this subfamily. The branching pattern of the phylogenetic tree indicates that mouse Skint1 to Skint6 genes emerged by rodent-specific gene duplication from an SKINT1L gene. Therefore, non-rodents do not have an SKINT gene orthologs to mouse Skint1. Actually, a gene ortholog to mouse Skint1 exists only in some rodents, specifically family Muridae or murids such as mice, rats, hamsters, and Mongolian gerbils. Coupled with the finding that Skint paralogs in mice have undergone functional specialization and have distinct functions $(22,25)$, these observations indicate that authentic Skint1 genes are unique to rodents, more precisely murids.

A notable feature of SKINT1L genes is that they are absent in a number of species such as elephants, sloths, armadillos, alpacas, horses, cats, dogs, and ferrets. They are also inactivated in several mammalian species. Thus, all the hominoids including humans, great apes (chimpanzees, gorillas, and orangutans), and lesser apes (gibbons) have SKINT1L genes inactivated by multiple mutations (29). One of the mutations, the stop codon located at the ninth residue of the $\mathrm{IgV}$ domain, is shared by all the hominoid sequences. Because Old World monkeys such as olive baboons, green monkeys, cynomolgus macaques, and rhesus macaques do not have this mutation, and their SKINT1L genes are apparently functional, this stop codon was most likely responsible for the initial inactivation of the hominoid SKINT1L gene (29). Tarsiers, pigs, 


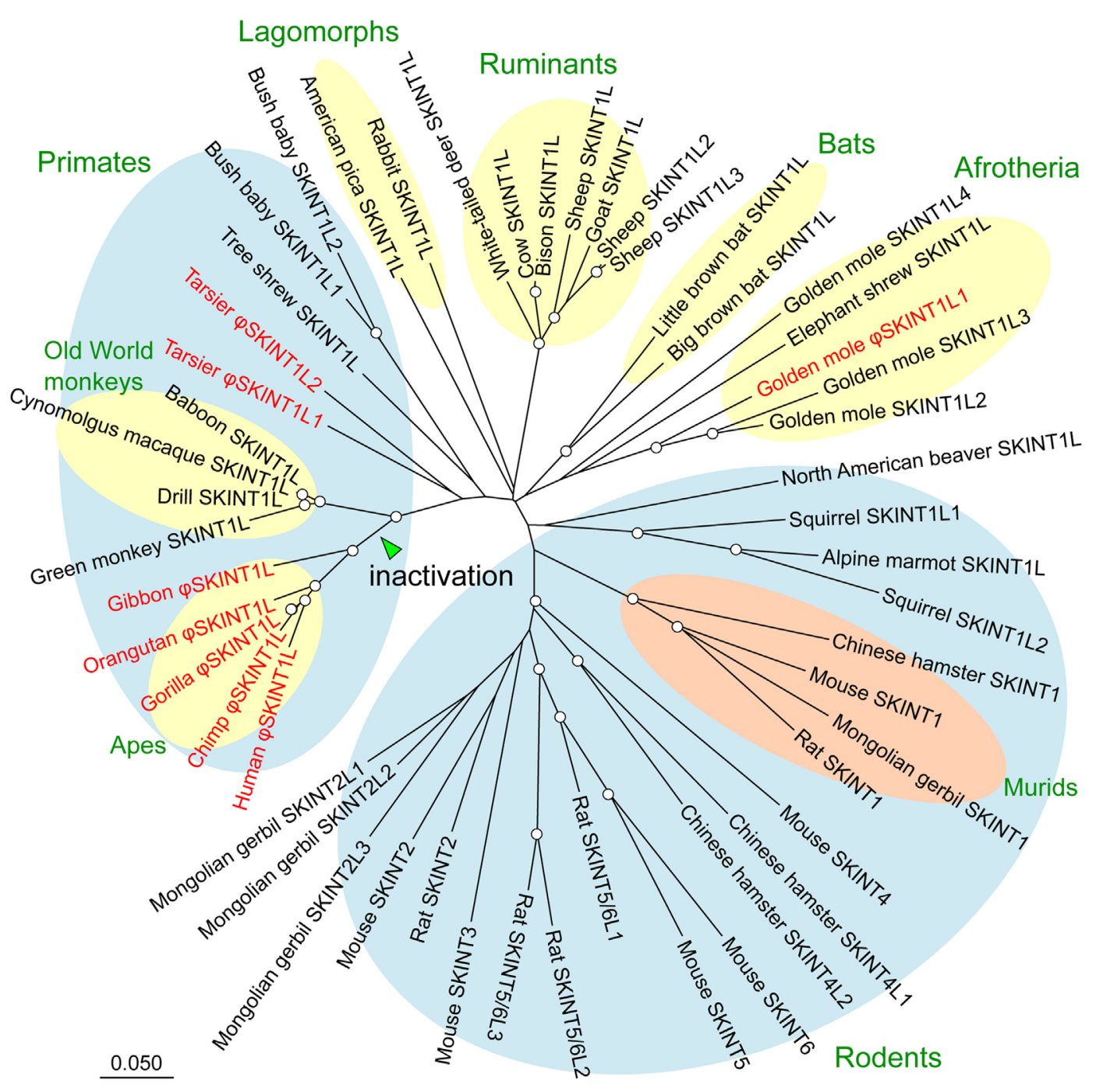

FIGURE 1 | Phylogenetic tree of the SKINT1 subfamily. SKINT1 subfamily sequences were retrieved from the NCBI database (WGS and nr databases) using the mouse Skint1 sequence as a query. Deduced amino acid sequences of SKINT1 subfamily proteins were aligned, and the phylogenetic tree was constructed using the MUSCLE and neighbor-joining programs implemented in MEGA7, respectively. The distance matrix was obtained by calculating p-distances for all pairs of sequences. Gaps were excluded using the pairwise-deletion option. The reliability of branching patterns was assessed by bootstrap analysis (1,000 replications). Pseudogenes are indicated by $\varphi$ and shown in red. Nodes supported by bootstrap values over $80 \%$ are indicated by open circles. Skint7 to Skint11 genes of mice are not shown as they are the members of the SKINT7 or SKINT9 subfamily.

and whales also have inactivated SKINT1L genes. Therefore, SKINT1L appears to have been lost or inactivated independently in multiple mammalian lineages.

\section{DETCS IN OTHER MAMMALS}

The observation that the Skint1 gene essential for DETC development exists only in rodents (Figure 1) indicates that Skint1dependent DETCs are unique to rodents. Indeed, rats are the only species in which the presence of cells quite similar to mouse DETCs has been unambiguously demonstrated (20). In the rat epidermis, $\alpha \beta$ T cells occupy only $0.03-0.24 \%$ of $\mathrm{CD}^{+}$cells, indicating that the vast majority of T cells are $\gamma \delta$ T cells (30). Immunostaining with a specific antibody against $\gamma \delta$ TCR revealed that the rat epidermis abundantly contains $\gamma \delta$ T cells with dendritic morphology (19). $\mathrm{V} \gamma$ and $\mathrm{V} \delta$ chains expressed on these $\gamma \delta \mathrm{T}$ cells are very similar to mouse $\mathrm{V} \gamma 5$ and $\mathrm{V} \delta 1$, with 92 and $95 \%$ amino acids sequence identity, respectively, and lack junctional diversity.

In cattle, a representative member of $\gamma \delta$-high species, more than $80 \%$ of skin T cells, of which at least $44 \%$ are $\gamma \delta \mathrm{T}$ cells, occur in the superficial $0.5 \mathrm{~mm}$ of the dermis, with only $3 \%$ in the epidermis (31). Thus, distribution of skin T cells differs from that in mice. Skin-resident bovine T cells are irregular in shape and frequently have a flattened outline with wavy cytoplasmic projections. Furthermore, although the information on TCR $\gamma$ and $\delta$-chain usage in epidermal $\gamma \delta$ T cells is not available, skinresident $\gamma \delta \mathrm{T}$ cells as a whole predominantly use $\mathrm{V} \gamma 3$ and $\mathrm{V} \gamma 7$ while the V $\delta$ usage is diverse (32). Therefore, it is possible that 
cattle have DETCs broadly defined as dendritic-shaped epidermal $\gamma \delta \mathrm{T}$ cells with limited antigen receptor diversity.

In humans, V $\delta 1 \mathrm{~T}$ cells, which express different Vy elements, are the major $\gamma \delta$ T cell subset preferentially homing to epithelial tissues such as skin and intestine $(33,34)$. In the skin, V81 T cells reside mainly in the dermis but are also found in the epidermis. Like mouse DETCs, activated V $\delta 1 \mathrm{~T}$ cells produce insulin-like growth factor 1 and promote wound healing (33). They also exert cytotoxic responses against tumors. Therefore, they seem to perform functions similar to those of rodent DETCs. However, because human epidermal V $\delta 1 \mathrm{~T}$ cells do not have a distinctive dendritic shape, it seems inappropriate to call them DETCs.

In non-human primates, the possibility of the existence of DETCs was examined in cynomolgus macaques (crab-eating macaques) because, unlike humans which have inactivated SKINT1L, macaques have a single copy of structurally intact SKINT1L (29). Like its mouse counterpart, macaque SKINT1L is expressed in the thymus and skin, and the basal and suprabasal layers of the macaque epidermis contain a population of dendritic-shaped $\gamma \delta \mathrm{T}$ cells. Macaque epidermal T cells predominantly expressed $\mathrm{V} \gamma 10 \mathrm{~V} \delta 1 \mathrm{TCRs}$, but both $\mathrm{V} \gamma$ and $\mathrm{V} \delta$ chains displayed junctional diversity. Also, expression of macaque $V \gamma 10$ was not restricted to epidermal lymphocytes. Therefore, it was concluded that macaques do not have rodent-type DETCs (29), but it is possible that they have DETCs defined as dendritic-shaped epidermal $\gamma \delta$ $\mathrm{T}$ cells with limited antigen receptor diversity.

In summary, DETCs that are selected by SKINT1 molecules and display an invariant $\gamma \delta$ TCR are unique to rodents, but DETCs in a broad sense appear to occur in other mammals, although more detailed investigation is required to draw definitive conclusions.

\section{ORIGIN AND EVOLUTION OF DETCs}

Recent work has uncovered that the epidermis of lampreys, a member of jawless vertebrates, contains dendritic-shaped $\gamma \delta$ T-like cells with limited antigen receptor diversity reminiscent of DETCs (35).

Jawless vertebrates represented by lampreys and hagfish are the most primitive class of vertebrates equipped with adaptive immunity; accumulated evidence indicates that lymphocytes forming the cornerstone of adaptive immunity emerged in a common ancestor of jawed and jawless vertebrates. Interestingly, instead of TCRs and B-cell receptors (BCRs), jawless vertebrates use members of the leucine-rich repeat (LRR) family of proteins known as variable lymphocyte receptors (VLRs) for antigen recognition (36-39). Like gnathostome antigen receptors, VLRs are clonally expressed on lymphocytes. The diversity of VLRs,

\section{REFERENCES}

1. Nielsen MM, Witherden DA, Havran WL. $\gamma \delta \mathrm{T}$ cells in homeostasis and host defence of epithelial barrier tissues. Nat Rev Immunol (2017) 17:733-45. doi:10.1038/nri.2017.101

2. Havran WL, Jameson JM. Epidermal T cells and wound healing. J Immunol (2010) 184:5423-8. doi:10.4049/jimmunol.0902733

3. Garman RD, Doherty PJ, Raulet DH. Diversity, rearrangement, and expression of murine T cell $\gamma$ genes. Cell (1986) 45:733-42. doi:10.1016/00928674(86)90787-7 which is assumed to be comparable to that of TCRs and BCRs, is generated during lymphocyte development by assembly of multiple LRR modules with highly variable sequences through a geneconversion-like mechanism. Jawless vertebrates have three major populations of lymphocytes distinguished by expression of distinct types of VLRs known as VLRA, VLRB, and VLRC. VLRB ${ }^{+}$cells resemble gnathostome $\mathrm{B}$ cells; when activated by specific antigen, they undergo clonal proliferation and secrete VLRB molecules as antibodies. On the other hand, $\mathrm{VLRA}^{+}$and $\mathrm{VLRC}^{+}$cells develop in lympho-epithelial thymus-like structures named thymoids, do not secrete VLR molecules, and resemble gnathostome T cells in gene expression profiles and responses to mitogens. Of the two T-like lymphocyte populations, $\mathrm{VLRC}^{+}$cells resemble $\gamma \delta \mathrm{T}$ cells in gene expression profiles in that they express the SRY-box containing gene 13 encoding a fate-determining transcription factor important for $\gamma \delta$ T-cell lineage determination and interleukin-17. They also resemble gnathostome $\gamma \delta$ T cells in tissue localization; $\mathrm{VLRC}^{+}$cells in lampreys are distributed predominantly in the epithelium of skin, intestine, and gill (35). In the epidermis, $\mathrm{VLRC}^{+}$cells are $\sim 8$ times more abundant than $\mathrm{VLRA}^{+}$cells and display dendritic morphology. Furthermore, the diversity of VLRC receptors in epidermal lymphocytes is markedly reduced compared to that in kidneys and blood.

The existence of DETC-like lymphocytes in the epidermis of lampreys indicates that the strategy of deploying $\gamma \delta$-like T cells to epithelia was adopted in a vertebrate ancestor and has been maintained in many vertebrate animals.

\section{CONCLUDING REMARKS}

Skint1-dependent DETCs appear unique to rodents such as mice and rats. However, DETCs broadly defined as dendritic-shaped epidermal $\gamma \delta$ T cells with limited antigen receptor diversity appear to exist in other mammals. The presence of DETC-like cells in lampreys suggests that DETCs also occur in jawed vertebrates other than mammals. In animals in which Skint1 is absent, other members of the butyrophilin family may perform equivalent functions. Also, "stress antigens" recognized by DETCs most likely differ from species to species. This difference, along with the difference in the butyrophilin members used for selection, may account for the observation that $\gamma \delta$ TCRs on DETCs are invariant in rodents, whereas those on putative DETCs in other animals are not invariant, but display limited diversity.

\section{AUTHOR CONTRIBUTIONS}

YS and RM conducted experiments which formed part of the arguments made in this paper. YS and MK wrote the paper.

4. Havran WL, Chien YH, Allison JP. Recognition of self antigens by skinderived T cells with invariant $\gamma \delta$ antigen receptors. Science (1991) 252:1430-2. doi:10.1126/science.1828619

5. Witherden DA, Verdino P, Rieder SE, Garijo O, Mills RE, Teyton L, et al. The junctional adhesion molecule JAML is a costimulatory receptor for epithelial $\gamma \delta$ T cell activation. Science (2010) 329:1205-10. doi:10.1126/science.1192698

6. Witherden DA, Watanabe M, Garijo O, Rieder SE, Sarkisyan G, Cronin SJ, et al. The CD100 receptor interacts with its plexin B2 ligand to regulate epidermal $\gamma \delta \mathrm{T}$ cell function. Immunity (2012) 37:314-25. doi:10.1016/j. immuni.2012.05.026 
7. Eagle RA, Trowsdale J. Promiscuity and the single receptor: NKG2D. Nat Rev Immunol (2007) 7:737-44. doi:10.1038/nri2144

8. Raulet DH, Gasser S, Gowen BG, Deng W, Jung H. Regulation of ligands for the NKG2D activating receptor. Annu Rev Immunol (2013) 31:413-41. doi:10.1146/annurev-immunol-032712-095951

9. Carapito R, Bahram S. Genetics, genomics, and evolutionary biology of NKG2D ligands. Immunol Rev (2015) 267:88-116. doi:10.1111/imr.12328

10. Kasahara M, Sutoh Y. Comparative genomics of the NKG2D ligand gene family. Immunol Rev (2015) 267:72-87. doi:10.1111/imr.12320

11. Sharp LL, Jameson JM, Cauvi G, Havran WL. Dendritic epidermal T cells regulate skin homeostasis through local production of insulin-like growth factor 1. Nat Immunol (2005) 6:73-9. doi:10.1038/ni1152

12. MacLeod AS, Hemmers S, Garijo O, Chabod M, Mowen K, Witherden DA, et al. Dendritic epidermal T cells regulate skin antimicrobial barrier function. J Clin Invest (2013) 123:4364-74. doi:10.1172/JCI70064

13. Lai Y, Li D, Li C, Muehleisen B, Radek KA, Park HJ, et al. The antimicrobial protein REG3A regulates keratinocyte proliferation and differentiation after skin injury. Immunity (2012) 37:74-84. doi:10.1016/j.immuni.2012.04.010

14. Havran WL, Allison JP. Developmentally ordered appearance of thymocytes expressing different T-cell antigen receptors. Nature (1988) 335:443-5. doi:10.1038/335443a0

15. Ito K, Bonneville M, Takagaki Y, Nakanishi N, Kanagawa O, Krecko EG, et al. Different $\gamma \delta$ T-cell receptors are expressed on thymocytes at different stages of development. Proc Natl Acad Sci U S A (1989) 86:631-5. doi:10.1073/ pnas.86.2.631

16. Aono A, Enomoto H, Yoshida N, Yoshizaki K, Kishimoto T, Komori T. Forced expression of terminal deoxynucleotidyl transferase in fetal thymus resulted in a decrease in gammadelta $\mathrm{T}$ cells and random dissemination of $\mathrm{V} \gamma 3 \mathrm{~V} \delta 1$ $\mathrm{T}$ cells in skin of newborn but not adult mice. Immunology (2000) 99:489-97. doi:10.1046/j.1365-2567.2000.00987.x

17. Jameson JM, Cauvi G, Witherden DA, Havran WL. A keratinocyte-responsive $\gamma \delta$ TCR is necessary for dendritic epidermal T cell activation by damaged keratinocytes and maintenance in the epidermis. J Immunol (2004) 172:3573-9. doi:10.4049/jimmunol.172.6.3573

18. Jameson J, Havran WL. Skin $\gamma \delta$ T-cell functions in homeostasis and wound healing. Immunol Rev (2007) 215:114-22. doi:10.1111/j.1600-065X.2006.00483.x

19. Kühnlein P, Park JH, Herrmann T, Elbe A, Hunig T. Identification and characterization of rat $\gamma / \delta \mathrm{T}$ lymphocytes in peripheral lymphoid organs, small intestine, and skin with a monoclonal antibody to a constant determinant of the $\gamma / \delta$ T cell receptor. J Immunol (1994) 153:979-86.

20. Kühnlein P, Mitnacht R, Torres-Nagel NE, Herrmann T, Elbe A, Hünig T. The canonical $\mathrm{T}$ cell receptor of dendritic epidermal $\gamma \delta \mathrm{T}$ cells is highly conserved between rats and mice. Eur J Immunol (1996) 26:3092-7. doi:10.1002/eji. 1830261240

21. Boyden LM, Lewis JM, Barbee SD, Bas A, Girardi M, Hayday AC, et al. Skint1, the prototype of a newly identified immunoglobulin superfamily gene cluster, positively selects epidermal $\gamma \delta$ T cells. Nat Genet (2008) 40:656-62. doi:10.1038/ng. 108

22. Barbee SD, Woodward MJ, Turchinovich G, Mention JJ, Lewis JM, Boyden LM, et al. Skint-1 is a highly specific, unique selecting component for epidermal Tcells. ProcNatlAcad SciUSA (2011) 108:3330-5. doi:10.1073/pnas.1010890108

23. Lewis JM, Girardi M, Roberts SJ, Barbee SD, Hayday AC, Tigelaar RE. Selection of the cutaneous intraepithelial $\gamma \delta+\mathrm{T}$ cell repertoire by a thymic stromal determinant. Nat Immunol (2006) 7:843-50. doi:10.1038/ni1363

24. Salim M, Knowles TJ, Hart R, Mohammed F, Woodward MJ, Willcox CR, et al. Characterization of a putative receptor binding surface on Skint-1, a critical determinant of dendritic epidermal $\mathrm{T}$ cell selection. J Biol Chem (2016) 291:9310-21. doi:10.1074/jbc.M116.722066
25. Keyes BE, Liu S, Asare A, Naik S, Levorse J, Polak L, et al. Impaired epidermal to dendritic T cell signaling slows wound repair in aged skin. Cell (2016) 167(1323-38):e14. doi:10.1016/j.cell.2016.10.052

26. Abeler-Dorner L, Swamy M, Williams G, Hayday AC, Bas A. Butyrophilins: an emerging family of immune regulators. Trends Immunol (2012) 33:34-41. doi:10.1016/j.it.2011.09.007

27. Afrache H, Gouret P, Ainouche S, Pontarotti P, Olive D. The butyrophilin (BTN) gene family: from milk fat to the regulation of the immune response. Immunogenetics (2012) 64:781-94. doi:10.1007/s00251-012-0619-z

28. Rhodes DA, Reith W, Trowsdale J. Regulation of immunity by butyrophilins. Annu RevImmunol (2016) 34:151-72. doi:10.1146/annurev-immunol-041015055435

29. Mohamed RH, Sutoh Y, Itoh Y, Otsuka N, Miyatake Y, Ogasawara K, et al. The SKINT1-like gene is inactivated in hominoids but not in all primate species: implications for the origin of dendritic epidermal T cells. PLoS One (2015) 10:e0123258. doi:10.1371/journal.pone.0123258

30. Elbe A, Kilgus O, Hunig T, Stingl G. T-cell receptor diversity in dendritic epidermal T cells in the rat. J Invest Dermatol (1994) 102:74-9. doi:10.1111/15231747.ep12371735

31. Hein WR, Dudler L. TCR $\gamma \delta+$ cells are prominent in normal bovine skin and express a diverse repertoire of antigen receptors. Immunology (1997) 91:58-64. doi:10.1046/j.1365-2567.1997.00224.x

32. Van Rhijn I, Rutten VP, Charleston B, Smits M, van Eden W, Koets AP. Massive, sustained $\gamma \delta \mathrm{T}$ cell migration from the bovine skin in vivo. J Leukoc Biol (2007) 81:968-73. doi:10.1189/jlb.0506331

33. Toulon A, Breton L, Taylor KR, Tenenhaus M, Bhavsar D, Lanigan C, et al. A role for human skin-resident T cells in wound healing. J Exp Med (2009) 206:743-50. doi:10.1084/jem.20081787

34. Pang DJ, Neves JF, Sumaria N, Pennington DJ. Understanding the complexity of $\gamma \delta$ T-cell subsets in mouse and human. Immunology (2012) 136:283-90. doi:10.1111/j.1365-2567.2012.03582.x

35. Hirano M, Guo P, McCurley N, Schorpp M, Das S, Boehm T, et al. Evolutionary implications of a third lymphocyte lineage in lampreys. Nature (2013) 501:435-8. doi:10.1038/nature12467

36. Pancer Z, Amemiya CT, Ehrhardt GR, Ceitlin J, Gartland GL, Cooper MD. Somatic diversification of variable lymphocyte receptors in the agnathan sea lamprey. Nature (2004) 430:174-80. doi:10.1038/nature02740

37. Flajnik MF, Kasahara M. Origin and evolution of the adaptive immune system: genetic events and selective pressures. Nat Rev Genet (2010) 11:47-59. doi: $10.1038 / \operatorname{nrg} 2703$

38. Boehm T, McCurley N, Sutoh Y, Schorpp M, Kasahara M, Cooper MD. VLRbased adaptive immunity. Annu Rev Immunol (2012) 30:203-20. doi:10.1146/ annurev-immunol-020711-075038

39. Kasahara M, Sutoh Y. Two forms of adaptive immunity in vertebrates: similarities and differences. Adv Immunol (2014) 122:59-90. doi:10.1016/ B978-0-12-800267-4.00002-X

Conflict of Interest Statement: The authors declare that this paper was written in the absence of any commercial or financial relationships that could be construed as a potential conflict of interest.

Copyright $\odot 2018$ Sutoh, Mohamed and Kasahara. This is an open-access article distributed under the terms of the Creative Commons Attribution License (CC BY). The use, distribution or reproduction in other forums is permitted, provided the original author(s) and the copyright owner are credited and that the original publication in this journal is cited, in accordance with accepted academic practice. No use, distribution or reproduction is permitted which does not comply with these terms. 